\title{
Manuel Granell: filósofo, discípulo y lector de Ortega. El camino fenomenológico de la Estimativa a la Ethología
}

\author{
Manuel Granell: philosopher, disciple and reader of Ortega. \\ The phenomenological path from Estimate to Ethology
}

\author{
NOÉ EXPÓSITO ROPERO* \\ IGNACIO QUEPONS RAMÍREZ**
}

\begin{abstract}
Resumen: Nuestro trabajo persigue cuatro objetivos. Primero, recuperar la figura filosófica de Manuel Granell (Oviedo, 1906 - Caracas, 1993), uno de los grandes discípulos de Ortega, olvidada en la actualidad. Segundo, mostrar que la recuperación del pensamiento de Granell nos ofrece algunas claves fundamentales para una lectura alternativa de la obra de Ortega a partir de la tradición fenomenológica inaugurada por Brentano y Husserl, distanciándose así, en este punto, de otros eminentes discípulos de Ortega, como Antonio Rodríguez Huéscar o Julián Marías. Tercero, abordar esta problemática al hilo de la ciencia Estimativa propuesta por Ortega en 1916, mostrando, en cuarto lugar, la estrecha relación entre ésta y la Ethología de Granell.
\end{abstract}

\begin{abstract}
The aim of our work is to address four points. First, to recover the philosophical figure of Manuel Granell (Oviedo, 1906 - Caracas, 1993), one of the great disciples of Ortega, unfortunately, forgotten. Second, to show that the recovery of Granell's work offers some keys for an alternative interpretation of Ortega's work considering the phenomenological tradition started by Brentano and Husserl, as a counterpart from the interpretation of other eminent disciples of Ortega like Antonio Rodríguez Huéscar or Julián Marías. Third, to address such problem following the Science of Estimation developed by Ortega in 1916, and forth, to show the close relation between such science and the so called "Ethologia" developed by Granell.
\end{abstract}

Recibido: 30/05/2019. Aceptado: 15/11/2019.

* Noé Expósito Ropero ha sido beneficiario del Programa de Formación de Profesor Universitario (FPU) 20152019 del Ministerio de Educación y Ciencia, adscrito al Departamento de Filosofía y Filosofía Moral y Política de la UNED, donde ha defendido su tesis doctoral sobre La ética fenomenológica de Husserl y Ortega: del deber al imperativo biográfico bajo la dirección del profesor Javier San Martín. Es miembro del proyecto de investigación "Fenomenología del cuerpo y análisis del dolor II" (FFI 2017-82272-P), dirigido por el doctor Agustín Serrano de Haro. Es autor, entre otras publicaciones, de "Lecturas de Ortega. A propósito de su fenomenología de los valores y su Estimativa", Acta Mexicana de Fenomenología. Revista de Investigación filosófica y científica, 4, 2019,pp.57-102.Correo electrónico: nexposito@fsof.uned.es

** Ignacio Quepons, investigador titular de tiempo completo, Instituto de Filosofía, Universidad Veracruzana, México.iquepons@uv.mx. Su investigación se orienta por el estudio de la dimensión afectiva y práctica de la subjetividad en la fenomenología trascendental, así como la relación entre Husserl con la historia de la filosofía. Recientemente trabaja en un análisis fenomenológico de la experiencia de la vulnerabilidad y la violencia social. Es editor del volumen Phenomenology of Emotions: historical and systematical perspectives, New Yearbook for Phenomenology and Phenomenological Philosophy, Routledge, 2018. 
Palabras clave: Manuel Granell; José Ortega y Gasset; Escuela de Madrid; Teoría del valor; Ética de la vocación; Fenomenología.
Key words: Manuel Granell, José Ortega y Gasset, Escuela de Madrid, Madrid School, Value Theory, Vocational Ethics, Phenomenology.

\section{Planteamiento, objetivos y contexto histórico-filosófico}

En un volumen monográfico sobre «El legado de Ortega y Gasset en el exilio republicano del 39. Continuidades y rupturas» habría que destacar -y rescatar del olvido- un gran número de figuras filosóficas, de mayor o menor relevancia, pero si hay una que merezca hoy toda nuestra atención, esa es, sin duda, la de Manuel Granell (Oviedo, 1906 - Caracas, 1993), incomprensiblemente olvidada en la actualidad. ${ }^{1}$ Podría discutirse si Granell forma parte o no del “exilio del 39", y autores como José Luis Abellán lo han negado expresamente (1978, 130), si bien, por otro lado, lo incluyó posteriormente, dedicándole algunas páginas en su libro sobre El exilio filosófico en América. Los transterrados de 1939 (1998, 179-193). Tales páginas son, dicho sea de paso, de las pocas que desde su muerte en 1993 se han escrito sobre el filósofo ovetense transterrado en Venezuela a partir de 1950. Por tanto, sin entrar aquí a discutir la cuestión -más historiográfica que filosófica- de si fue Granell o no un "exiliado del 39", ya que él mismo reconoció en una entrevista en 1980 que su marcha a Caracas tuvo también un componente de "elección"2, lo que resulta incuestionable es que su obra se proyecta en la estela de los discípulos de Ortega de dicha generación. En este sentido, y sin entrar, para decirlo con Ortega, en la consistencia de la denominada "Escuela de Madrid" -término acuñado originalmente por Granell en $1953^{-3}$, ni tampoco en quiénes la conformaban en sus inicios ${ }^{4}$, lo que

1 Y esto a pesar de que sus obras están disponibles en internet y son de acceso de público desde que la Fundación Manuel Granell las editara digitalmente en 2008, de modo que pueden descargarse en el siguiente enlace: http:// www.fundacionmanuelgranell.com/manuelGranell.swf [visitado por última vez el 21.5.2019]. En lo que sigue, si no se indica lo contrario, citaremos las obras de Granell por esta edición digital.

2 Véase la "Entrevista a Manuel Granell" realizada en 1980 por Luis Javier Álvarez y Alberto Hidalgo, donde afirma lo siguiente: "Dicho esto, me ciño al preguntar sobre mi caso: ¿exilio o elección? Ambas cosas, respondo, pues he sufrido ambos exilios, el material y el de conciencia" $(1980,56)$. Para más detalles sobre la biografía de Granell nos remitimos tanto a esta entrevista como al citado estudio de Abellán.

3 Hasta donde sabemos, y así nos lo recuerda José Lasaga (Lasaga, 2016: 121; San Martín, 2016: 260), fue Granell quien empleó por primera vez el rótulo "La Escuela de Madrid" en un artículo con ese mismo título, donde afirma, refiriéndose a la filosofía incipiente en la Facultad madrileña durante la República como "una misteriosa incitación” que, "desde ella, precisamente, pudiera resumirse lo más vivo y mejor del especial espíritu filosófico de la que me complazco en llamar Escuela de Madrid" (cursivas del propio Granell). Esta cita procede, insistimos, de un escrito titulado "La Escuela de Madrid", recogido posteriormente, como es sabido, en su libro Ortega y su filosofía (1959), pero el dato relevante nos lo frece Granell en nota al pie afirmando que este texto fue "Publicado en Caracas, Mayo de 1953, como homenaje a los setenta años del maestro Ortega. Se había escrito para un Congreso Internacional de Filosofía a celebrarse en La Habana, luego suspendido. Refiero el dato como justificación de su estilo" (Granell, 2008, 61, nota 42). El hecho de que el propio Granell escribiera "me complazco en llamar Escuela de Madrid" nos indica, muy presumiblemente, que no tenía noticia previa del empleo de tal rótulo, de ahí que se atribuya al filósofo ovetense esta primicia. Por nuestra parte, no hemos encontrado tal referencia en el escrito de Gaos al que nos remite Scotton cuando afirma que "La invención del lema Escuela de Madrid se debe muy probablemente a José Gaos que la utilizó por primera vez en un artículo de 1939" (Scotton, 2018, 498, nota 4).

4 Sobre esta y otras temáticas relacionadas con la "Escuela de Madrid" nos remitimos, entre otros, a los ya clásicos estudios de Marías (1959), Abellán (1978), Abellán y Mallo (1991), así como a los más recientes de Padilla 
sí nos parece innegable es que difícilmente podrá narrarse la historia del legado filosófico de Ortega en el "exilio del 39" sin atender a la figura de Granell. Y más aún cuando se trata de establecer las "continuidades y rupturas" de este legado.

En lo que sigue intentaremos justificar estas aseveraciones, persiguiendo, fundamentalmente, cuatro objetivos: primero, recuperar y contextualizar la figura filosófica de Manuel Granell; segundo, ofrecer una exposición crítica del pensamiento de Granell en relación con las claves fundamentales -según insistió el propio filósofo ovetense- de una alternativa de lectura del legado de Ortega en relación con su influencia e inserción en la tradición fenomenológica inaugurada por Franz Brentano y Edmund Husserl, frente a la interpretación de otros discípulos de Ortega, como Antonio Rodríguez Huéscar o Julián Marías.

En este sentido, nuestra tesis principal es que Granell resulta una figura clave para sopesar las "continuidades y rupturas" del legado orteguiano, ya que, continuando su legado, rompe, sin embargo, con una de las tesis nucleares de la interpretación de Marías, quien era, según Abellán, "el intérprete oficial de Ortega en España" (1978, 72). Tercero, mostrar la continuidad de Ortega con la fenomenología al hilo de la ciencia Estimativa que nos presenta en 1916 y desarrolla en escritos como el Discurso para la Real Academia de Ciencias Morales y Políticas de 1918 (inédito hasta 2007) o su "Introducción a una Estimativa: ¿qué son los valores" de 1923. ${ }^{5}$ La reivindicación de esta ciencia Estimativa, prácticamente olvidada por los intérpretes de Ortega hasta hoy, nos revelará aspectos fenomenológicos de la filosofía orteguiana que resultan imprescindibles para la formulación de conceptos tan decisivos como el de "ethos". Cuarto, mostrar la intrínseca relación entre la Estimativa orteguiana y la Ethología propuesta por Granell como "la única ciencia que cuadraría al estudio del hombre", según sostuvo el filósofo ovetense. De este modo, la unidad de la interpretación que proponemos aquí descansa en destacar cómo el camino que va de la Estimativa de Ortega a la Ethología de Granell puede entenderse como un desarrollo original de la senda abierta por Husserl. A nuestro juicio, tanto Ortega como posteriormente Granell cosecharán enormes frutos, en medio de otras influencias, como la axiología de Scheler y la filosofía de Heidegger, cuya raíz se encuentra en última instancia en los ejes cardinales de la ética y la teoría del valor, menos estudiados, planteada entre 1908 y 1914 por el fundador del movimiento fenomenológico: Edmund Husserl. ${ }^{6}$

Así, en lo que sigue dividiremos nuestro trabajo en tres apartados. En el primero nos ocuparemos de Granell como filósofo, intentando ofrecer una síntesis de las líneas generales de su propio proyecto intelectual. En el segundo incidiremos en la importancia de Granell en tanto que discípulo de Ortega, mostrando algunas continuidades y rupturas en relación con el legado orteguiano tal y como ha sido transmitido "oficialmente" por otros discípulos

(2007), García Gómez (2009), o los recogidos en el Boletín de estudios de filosofía y cultura Manuel Mindán de 2011 dedicado a La Escuela de Madrid.

5 Recogidos en los tomos VII y III de las Obras completas, 10 vols. Fundación José Ortega y Gasset/ Taurus, Madrid, 2004-2010, pp. 703-738 y 531-549 respectivamente. En lo que sigue incluiremos las referencias a las Obras completas de Ortega en el cuerpo textual principal indicando, como es habitual, el volumen en números romanos y las páginas en arábigos.

6 Puesto que un desarrollo minucioso de estas líneas de investigación desbordaría los límites de nuestro trabajo, nos limitamos en lo que sigue a esbozar los que serían, a nuestro juicio, los puntos más importantes a tratar. Con todo, consideramos que estas cuatro líneas temáticas están intrínsecamente relacionadas, de ahí la pertinencia de presentarlas conjuntamente en un mismo trabajo. 
directos, tales como Marías o Rodríguez Huéscar. En tercer lugar, destacaremos la relevancia de la lectura de Ortega que nos ofrece Granell, ya que, a nuestro juicio, con ella se inicia la lectura fenomenológica desarrollada posteriormente por Javier San Martín, en la que enmarcamos nuestro propio trabajo. Finalmente, a modo de conclusión, esbozaremos algunas reflexiones en torno a todas estas problemáticas.

\section{Manuel Granell, filósofo. Humanismo integral y Ethología}

El primer hecho que llama la atención a quienes nos hemos dedicado mínimamente al estudio de la obra de Manuel Granell quizás sea, antes que nada, la escasa atención que el filósofo ovetense ha merecido entre nuestros contemporáneos. No se comprende cómo una obra de la densidad y complejidad de La vecindad humana. Fundamentación de la Ethología (1969), publicada nada menos que en Revista de Occidente, ha pasado prácticamente desapercibida hasta nuestros días. Esta es, según nos dice retrospectivamente el propio Granell en Ethología y existencia (1977), su "obra más cabal", advirtiéndonos expresamente que el resto de sus escritos "giran en torno a ella, unas veces como introducción, otras a manera de complemento, siempre en búsqueda de verificación y de precisiones" (Granell, 2008, 31). Por tanto, insiste Granell, sus escritos se nos ofrecen, en cierta medida, como "calas diversas de lo mismo, aunque realizadas en diferentes ámbitos humanos -pues la ethología tiende de suyo a fundamentar todas las ciencias humanísticas" (ib.). Tal es, pues, la primera aproximación que cabría hacer al filósofo Manuel Granell, es decir, a su propia obra filosófica, con independencia de su condición discipular de Ortega. Sin embargo, hasta donde hemos podido investigar, carecemos todavía de nada parecido a un estudio más o menos crítico o sistemático de su obra. Tras las citadas páginas que Abellán le dedica en su libro de 1998, parece que solo Paolo Scotton se ha ocupado con cierto detenimiento de nuestro filósofo, ofreciéndonos una panorámica general de su filosofía en su reciente estudio titulado "La formación del ser humano. Sobre el humanismo de Manuel Granell” (2018). Scotton insiste, sobre todo, en la dimensión humanista de su pensamiento, pues, efectivamente, el propio Granell nos dice en Ethología y existencia que su posición filosófica cabría "filiarla bajo una etiqueta. Propondría: Humanismo integral. Humanismo a todo lo ancho y de abajo arriba; el ente biológico llamado hombre como artífice exclusivo de lo humano. De otro modo: evolución segunda. Con acento más propio: fundamentación ethológica" $(2008,10)$. No es de extrañar, pues, que su última antología filosófica publicada llevara por título, precisamente, Humanismo integral (1983).

Análogamente enfatiza Scotton, acertadamente a nuestro juicio, la dimensión "pedagógica" del pensamiento de Granell -por más, también ha de advertirse, que el término "pedagogía" apenas aparezca en el conjunto de su obra. Por ello, sin entrar a discutir aquí la tesis de Scotton según la cual "para Granell la ethología sería una extensión sustantiva de la pedagogía en cuanto ciencia formadora del hombre" (2018, 506, nota 32), sí que nos parece que para comprender a fondo su proyecto filosófico hemos de atender, según la propia cita de La vecindad humana que toma Scotton para mostrar su tesis (cfr. ib.), a la dimensión ontológica del mismo. Y esto porque, como afirma allí Granell, la ethología "aspira a ser técnica reformadora del hombre mismo desde su originaria raíz ontológica. No apunta, pues, a los individuos de carne y hueso, al menos directamente, sino a la viva nostridad del ethos-morada”, concibiéndola, prosigue, como "Técnica de segundo grado, fautora o protectora de esta técnica originaria del 
vivir, la ethología pretende llegar con su escalpelo hasta el pálpito cordial de la ontológica superación" (cfr. Granell, ib., 576-577). Advertimos, pues, y esto es lo decisivo, cómo la obra de Granell merece y exige por sí misma una detenida confrontación filosófica, más allá de su condición discipular de Ortega, de ahí que la primera reivindicación que pretendemos hacer en nuestro trabajo sea, precisamente, la recuperación de Granell como filósofo con nombre propio y una obra original. El propio Granell nos ofrece en Ethología y existencia (1977) una magnífica síntesis retrospectiva de su posición filosófica, de modo que merece la pena reproducir aquí el siguiente pasaje:

Naturalmente, estoy iluminando con luces actuales el viejo sentir. Hasta muchos años después no logré apresar con plenitud mi oscuro afán. Hoy puedo definirlo -contra el pretendido axioma- con estas palabras: la realidad es tercera. Y lo es, precisamente por derivarse del hecho radical que nos enmarca: la "ex-sistencia". El detenido análisis de ésta -desde el cual arranca mi construcción filosófica- pone al aire dos instancias contrapuestas, en constante lucha, la "in-sistencial" y la "re-sistencial", pero con tan cambiado y alternado ejercicio, que todo funciona en cierto "entre" de sí mismas, pues aparecen en mutua inmanencia, una dentro de la otra, pasándose -podría decirse- al enemigo. El humano ímpetu creador de hoy deviene pétrea resistencia mañana, justo porque hubo de obyectarse fuera del hombre para cobrar vida en su ahí. El "ahí-mostrenco" -donde el ethos mora sobre corrientes sintagmáticas- se constituye, en efecto, como un desembocar desde instancias pugnantes y en base al conjugado esfuerzo del "aquí-propio" y del "alli-vocado". Sólo cabe mencionar en estas líneas esos tres existenciarios estructurales y los conceptos anexos, que el lector verá más adelante, y sobre todo en La vecindad humana" (ib., 12).

A estos escritos nos remitimos, pues, para una mayor profundización en la obra filosófica de Manuel Granell, donde encontraremos el desarrollo de las líneas generales de su pensamiento que hemos intentado sintetizar en las líneas precedentes.

\section{Manuel Granell, discípulo de Ortega}

En segundo lugar, y no de menor importancia, Granell nos interesa también por ser, efectivamente, uno de los más eminentes discípulos de Ortega. Y esto, dicho sea de paso, por más que su lectura del texto orteguiano haya quedado ensombrecida por la que ha sido, hasta la fecha, la "interpretación oficial" de Ortega mantenida y transmitida, fundamentalmente, por Julián Marías y Antonio Rodríguez Huéscar. ${ }^{7}$ Es de sobra conocida la afirmación orteguiana, expuesta retrospectivamente en La idea de principio en Leibniz (1947), según la cual, escribe nuestro filósofo, "abandoné la Fenomenología en el momento mismo de recibirla" (IX, 1119). Como sabemos, la "lectura oficial" de Ortega asumió completamente esta afirmación, de ahí que Marías la intentara justificar en su trabajo de 1957, "Conciencia y realidad

7 En otro trabajo hemos intentado distinguir y clasificar las distintas "lecturas de Ortega" que los intérpretes han mantenido desde su muerte hasta la actualidad, incidiendo en la problemática relación de Ortega con la fenomenología (cfr. Expósito Ropero, 2019), pues ya sabemos la compleja recepción de Husserl en el ámbito hispanohablante (cfr. Serrano de Haro, 2009; Díaz Álvarez, 2009). 
ejecutiva. La primera superación orteguiana de la fenomenología”, recogido, primero, en $L a$ Escuela de Madrid (1959, 255-264), y luego en su libro Acera de Ortega (1971, 139-147). La misma tesis encontramos en el "Prólogo" " que el filósofo vallisoletano escribió en 1982 para el libro de Rodríguez Huéscar sobre La innovación metafísica de Ortega:

La obra de Husserl significa el inicio de nuestro tiempo. Y resulta que en el seno de este amanecer rebrota, en forma de idealismo fenomenológico, el error que ha corroído todo el pensamiento de la modernidad, el que ha impedido dejar atrás definitivamente las limitaciones del realismo (Marías, 1982, 13).

Y, análogamente, tal es la postura que asume Rodríguez Huéscar en el citado libro, para quien "el tramo final del idealismo", escribe, "estaba representado por Husserl y su fenomenología", viendo en ella su último "canto de cisne" (cfr. 1982, 19; 77). Sin embargo, esta "interpretación oficial", adoptando la expresión de Abellán, es puesta en duda polémicamente -puesto que suponía revisar críticamente las afirmaciones del propio Ortega respecto a su relación con la fenomenología- en los años 70, ${ }^{9}$ y, ya definitivamente, en 1984 con el libro de Pedro Cerezo La voluntad de aventura, donde, como es sabido, califica a las lecturas de Marías y Rodríguez Huéscar a este respecto de "hermenéutica venerativa" $(1984,12 ; 226$, nota $97 ; 268$, nota 28$)$. En este contexto, ha sido Javier San Martín quien ha defendido abiertamente, ya desde los años 90 , que "no se debe hablar de la fenomenología EN Ortega, como si fuera esta una influencia más de las muchas que actúan en él, sino que la fenomenología es LA FILOSOFÍA DE Ortega", según mantiene en su último libro dedicado a esta temática, La fenomenología de Ortega y Gasset $(2012,13)$. Advertimos, pues, cómo el debate de fondo gira en torno a la cuestión central de cuál sea la filosofía de Ortega, lo cual resulta, obviamente, de un larguísimo alcance.

Pues bien, la tesis que mantenemos es que, frente a la "interpretación oficial" de Marías y Rodríguez Huéscar, Granell nos ofrecería una lectura alternativa del texto orteguiano, en clave fenomenológica, puesto que, a diferencia de sus colegas, el filósofo ovetense parece que nunca terminó de creer -o no, al menos, con la fe del carbonero, si se nos permite la expresión- que Ortega abandonara o superara tan prontamente la fenomenología de Husserl. Y, desde luego, no encontramos en sus escritos nada parecido a las críticas tópicas asumidas por Marías y Rodríguez Huéscar, tales como las expuestas, sino, más bien, un constante diálogo crítico con la obra husserliana, de la que Granell se nos revela un ávido y perspicaz lector. Merece la pena recordar aquí algunas consideraciones retrospectivas del filósofo ovetense para dar cuenta de lo que pretendemos mostrar. En Ethología y existencia relata Granell lo siguiente refiriéndose al magisterio de Ortega en sus años de formación universitaria:

El nombre que en Madrid resumía "la" filosofía era el del primer Husserl. Entonces no se sospechaba siquiera su posterior arrepentimiento. Y con Husserl, el primer

8 Como es sabido, este "Prólogo" no fue incluido en la reedición que del mismo libro se publicó en 2002, siendo sustituido por una "Semblanza de Antonio Rodríguez Huéscar" firmada por Javier Muguerza (2002, 11-16).

9 Cfr. San Martín, J. (2012), especialmente el capítulo 1. "La fenomenología como llave para entender a Ortega", pp. 17-50, donde nos ofrece un detallado análisis del estado de la cuestión desde los años 70 hasta la actualidad. 
Scheler, algo del primer Heidegger -tan diversos al segundo Scheler, al segundo Heidegger, y estos a su vez tan opuestos entre sí-. Al fondo del escenario, a veces ante las candilejas, otras dos figuras: Brentano y Hartmann. Todo se iluminaba bajo esta luz: fenomenología. Las Investigaciones lógicas se estudiaban con Gaos al dedillo, analizando sus entresijos en cada página, desmontando el encaballamiento argumental. Me hallaba otra vez ante otro esfuerzo de pureza. Su distinguir y encajar lo lógico puro era apabullante. ¡Aquel ejemplo de la máquina de calcular! Y luego, las reducciones, la apodicticidad... Husserl me atraía y repelía de consuno. Su influjo me era tan fuerte como mi reacción. Cierta vez -muchos años más tarde, ya en Caracasconfesé a Gaos el propósito de escribir otras investigaciones lógicas sobre el modelo de las husserlianas, al modo que Leibniz hizo con Locke, para replicarle así paso a paso. Por fortuna, curó mi afán la simple confesión del propósito. En la Facultad madrileña resonaba por añadidura esta terrible frase: el absolutismo de los valores. Era sobrecogedor. Los valores planeaban majestuosos por el cielo sin mácula de Platón. Paradójicamente, los valores me daban cierto respiro. “Aquí abajo"..., me decía. No sin alguna trampa, podía acentuarse el estimar, galvanizando los valores. Pero el respiro máximo lo hallaba en las clases de Ortega (2008, 16, cursivas en el original).

Se advierte ahora la distinta concepción y apreciación que de la fenomenología nos ofrece Granell, matizando, además, que en la Facultad madrileña estudiaron al "primer" Husserl, pero él conocía bien, tal y como nos había recordado ya en La vecindad humana (1969), "la posición del segundo Husserl, el del Lebenswelt, e incluso el Husserl de los inéditos, el que habla de «realizar una razón» y sustituye la vieja apodicticidad -ahora calificada de abstracta- por un «télos apodíctico» (cf. Husserl, La filosofía como toma de conciencia de la humanidad)" (Granell, 2008, 203).

Aquí encontramos, pues, la primera "ruptura" importante de Granell con el "legado oficial” de Ortega representado por Marías y Rodríguez Huéscar. Este relato nos muestra a las claras la formación fenomenológica de los discípulos de Ortega, solo que algunos se quedaron, efectivamente, en el "primer" Husserl, asumiendo así las críticas del maestro, mientras que otros, como Granell, dieron un paso más en la dirección trazada por Ortega. Y este paso más se nos revela, precisamente, en la segunda parte de la cita, la referida a los valores y al estimar -expresiones que el propio Granell enfatiza en cursiva. Como señalábamos al comienzo, la ciencia Estimativa que Ortega presenta en público por primera vez en 1916 en las conferencias impartidas en Buenos Aires ha sido, salvo algunas notables excepciones, ${ }^{10}$ prácticamente desatendida por los intérpretes, y solo muy recientemente ha empezado a recuperarse gracias a la publicación en la Revista de Estudios Orteguianos de materiales inéditos como las "Notas de trabajo sobre Estimativa" editadas por Javier Echeverría y Dolores Sánchez entre 2016 y 2017. ${ }^{11}$ Recordemos, pues, lo que entiende Ortega por tal ciencia Estimativa en las citadas conferencias:

10 Algunas excepciones serían, efectivamente, Ignacio Sánchez Cámara (2010), Diego Gracia (2011), Javier San Martín (2013) y Jorge Acevedo (2015, 167-186).

11 De especial relevancia resulta, igualmente, el estudio de presentación de estos materiales, "La Estimativa de Ortega y sus circunstancias" (2017), firmado por Javier Echeverría y Sandra García. Para un estudio crítico 
Más allá de los objetos empieza el mundo de los valores; más allá que el mundo de lo que es y de lo que no es, el mundo de lo que vale y de lo que no vale, el mundo de la ética y el mundo de la estética. Todo ello reunido en una ciencia peculiar distinta de todas las demás, sería lo que yo propongo llamar ciencia estimativa (VII, 663).

Pues bien, la tesis que mantenemos aquí es que en ella encontramos, a nuestro juicio, el vaso comunicante que nos conduce a la Ethología de Granell. Y es que, como nos recuerda el filósofo ovetense, junto a la fenomenología, "en la Facultad madrileña resonaba por añadidura esta terrible frase: el absolutismo de los valores". Ahora bien, Granell advirtió muy perspicazmente que, por paradójico que pudiera parecer, el discurso fenomenológico sobre "los valores" nos mantenía "aquí abajo", añadiendo, eso sí, el siguiente matiz decisivo: "No sin alguna trampa, podía acentuarse el estimar, galvanizando los valores". ¿A qué trampa se refiere Granell?, ¿de qué modo se estaban galvanizando, esto es, cubriendo o recubriendo los valores mediante la acentuación del estimar? Para responder a esta pregunta tampoco carece de interés la frase que cierra la citada narración de Granell: "Pero el respiro máximo lo hallaba en las clases de Ortega". Conviene recodar en este preciso momento lo que nos dice Granell en la citada entrevista realizada en 1980, cuando, ante la pregunta de "cómo se inició su vocación filosófica", responde: "Se centra en el curso académico 1924-25, durante el cual viví en Madrid y que considero fundamental en mi vida" (1980,48). Pues bien, como sabemos, uno de los conceptos centrales en la filosofía de Granell es el de ethos, a partir del cual construye su Ethología. Este concepto aparece por primera vez en Ortega en un artículo publicado en 1926 en El Sol, cuyo título reza "Destinos diferentes" (II, 616-618), donde parece disculparse, incluso, por la introducción de tal recurso terminológico:

Siento emplear el vocablo ethos, que es demasiado académico para no ser desagradable. Pero urge inculcarlo en el uso banal, porque, de una parte, no es fácil sustituirlo, y de otra, se refiere a cuestiones sobre que cada día será forzoso hablar más. Entiendo por ethos, sencillamente, el sistema de reacciones morales que actúan en la espontaneidad de cada individuo, clase, pueblo, época. (II, 616, cursivas de Ortega).

Hasta ahora, este artículo de 1926 ha sido el que los intérpretes han tenido como referencia para el estudio de este concepto. Sin embargo, en el tomo VII publicado en 2007 encontramos un borrador titulado "[Militares y clases mercantiles]" que resulta decisivo para esta problemática. En primer lugar, porque es, sorprendentemente, en este manuscrito inédito donde Ortega se ocupa con mayor intensidad de este concepto, donde aparece hasta en 18 ocasiones en sus tres páginas y media, mientras que en el resto de las Obras completas solo tenemos noticia del concepto en siete ocasiones, la mayoría de pasada y sin más elaboración técnica. ${ }^{12}$ En segundo

de este trabajo cfr. Expósito Ropero, "La Estimativa de Ortega: de sus circunstancias a sus bases fenomenológicas" (2019b)..

12 Aparte de los dos textos citados, el conceptos solo aparece en los siguientes escritos: una vez en "La interpretación bélica de la historia" (II, 636); una vez en La rebelión de las masas (IV, 496); dos veces en "Prólogo a Veinte años de caza mayor, del conde de Yebes" (VI, 307); una vez en Papeles sobre Velázquez. (VI, 709); una vez en "[Apuntes para un comentario al Banquete de Platón]” (IX, 735, nota 1); una vez en De Europa meditatio quaedam $(\mathrm{X}, 117)$. 
lugar, y esto es lo decisivo, es en este manuscrito inédito, y no así en el publicado, donde Ortega vincula expresamente el concepto de ethos a su Estimativa, recordándonos que tal era el tema que por aquellos años estaba desarrollando en sus lecciones universitarias -a las que, precisamente, se refiere Granell. Dado que hasta hoy ha sido prácticamente desatendido por los intérpretes, ${ }^{13}$ y que resulta decisivo para el tema que nos ocupa, merece la pena reproducir el siguiente pasaje del texto "[Militares y clases mercantiles]":

\begin{abstract}
Ahora bien, eso que vagamente llamamos temperamento íntimo y que nuestros antepasados denominaban la índole del sujeto, consiste meramente en una estructura de simpatías y antipatías nativas, de preferencias y posposiciones, de estimaciones y repulsiones. Más allá del plano en que se mueven nuestras ideas operan ocultas nuestras personales valoraciones. Cada uno de nosotros es, en definitiva y antes que nada, un sistema de valoraciones, un preferir ciertas cosas y posponer otras, un amar esto y odiar aquello. En mis lecciones universitarias suelo llamar a esta primaria actividad de nuestro espíritu «función estimativa»; ella es la raíz de la persona y de ella depende la función intelectiva y la volitiva y cuantas pueda distinguir la psicología en nuestra vida mental. Conviene, para entendernos, dar algún nombre específico a ese «carácter estimativo»y, por ello, siguiendo la noble tradición del pensamiento griego le llamaremos ethos. Cada persona, pues, está primariamente constituida por un ethos individual, un sistema peculiar de amores y odios, de preferencias y negligencias. A su vez, un pueblo, una época, se caracterizan en última instancia por un ethos determinado. El derecho, la ciencia, la economía, el arte, de un pueblo o de una época dependen de su ethos (VII, 755).
\end{abstract}

Se comprende ahora la trampa a la que hacía alusión Granell al decirnos que, "No sin alguna trampa, podía acentuarse el estimar, galvanizando los valores". Tal era, con todo, "el respiro máximo" que el filósofo ovetense hallaba en las clases de Ortega. Creemos, pues, que no hay que arriesgar grandes hipótesis hermenéuticas para sostener la intrínseca relación entre la Estimativa orteguiana y la Ethología de Granell, materializada, por ejemplo, en el "Proyecto de investigación conjunta del ethos venezolano" que presenta en 1973 a los restantes Institutos de la Facultad de Humanidades en la Universidad Central de Venezuela, según queda esbozado en Ethología y Existencia (cfr. ib., 9-10; 177-186). Por lo demás, conviene recordar que el propio Ortega concebía la Estimativa, no como una mera ciencia

13 Una excepción sería, sin embargo, Parra Ferreras, quien alude a él en su Tesis Doctoral (2015, 76-78), aunque asume, con los editores de las Obras completas (cfr. VII, 900-901), que el escrito data de 1920. Los editores arguyen que, efectivamente, el comienzo de este borrador coincide con otro texto de ese mismo año, a saber, "Particularismo y acción directa" (cfr. III, 435 y VII, 754). Sin embargo, bien pudiera ser, como postulamos nosotros, que Ortega retomara en 1926 el artículo de 1920 para arrancar su escrito "[Militares y clases mercantiles]", por lo que este dataría de la primera fecha, y no de la segunda. Una mínima comparación entre este borrador y el publicado en 1926 donde aparece por vez primera el concepto de ethos, "Destinos diferentes", nos revela que Ortega trata en ambos los mismos temas a propósito de la situación política en Barcelona (cfr. II, 617 y VII, 754) -recuérdese el intento de golpe de Estado en junio de 1926, al que alude Ortega expresamente. Esta coincidencia temática y, sobre todo, que es en ambos textos donde encontramos, insistimos, la más detenida y profunda elaboración orteguiana del concepto de ethos, sobre todo en el manuscrito inédito, nos hace postular que este no era sino el borrador del escrito publicado en 1926, "Destinos diferentes". 
teórica, formal y abstracta, sino que ya en su Discurso para la Real Academia de Ciencias Morales y Políticas de 1918 (inédito también hasta 2007) afirma expresamente lo siguiente, ofreciéndonos un interesantísimo esquema programático que merece la pena reproducir para mostrar tanto el alcance como la intrínseca relación entre ambos proyectos:

Junto a la Estimativa que aspira a conocer la naturaleza objetiva de los valores y su jerarquía esencial, surge, como un problema aparte y distinto, de gran fecundidad para la historia y para todas las ciencias morales, el examen de las valoraciones humanas, de la evolución de la conciencia de los valores, de las condiciones reales que determinan las variaciones en nuestra perspectiva de estimación. Éste es el problema psicológico e histórico.

Lo primero que habría de hacerse sería la reconstrucción descriptiva, por decirlo así, la anatomía de los sistemas típicos de valoración que caracteriza las condiciones facticias de los sujetos. Así -y sirva como muestra de la abundancia de temas que la Estimativa trae a la futura investigación- habría que fijar los sistemas de valoración propios a:

a) Cada pueblo.

b) Cada etapa histórica (Edad Antigua, Media, Moderna, Contemporánea son, ante todo, modificaciones típicas en la valoración, perspectivas de valores).

c) Cada oficio y clase social.

d) Las grandes figuras cuya obra ha sido principalmente la invención de nuevos valores en la conducta humana (así Budha, Cristo, San Francisco de Asís, Napoleón, etcétera). e) Las grandes figuras que no han tenido un carácter específico de hombres «prácticos», esto es de moralistas, políticos, etcétera. Así, los grandes artistas y pensadores no moralistas ni políticos: Miguel Ángel, Goya, Cervantes, etcétera.

La lista de temas sería muy larga, aun sin salir del orden descriptivo. Frente a éste, sobrevienen las investigaciones genéticas en que se indagarán las causas o condiciones de la variación en los sistemas de valorar: los cambios estimativos en un pueblo, en una clase, en la humanidad culta entera, en un individuo, etcétera (VII, 733).

Estamos, pues, como muy bien advirtió Granell, ante un fecundísimo campo de investigación todavía por explorar. Recordemos que este esquema data de 1918, pero Ortega lo reprodujo, aunque no en forma esquemática, sino redactado, en el texto sobre Estimativa de 1923, donde añade, por ejemplo, las figuras de Dostoyewsky (sic.) o Stendhal, ${ }^{14}$ lo cual indica que nunca dejó de tenerlo presente durante el trascurso de estos años. Tampoco es, por lo demás, ninguna hipótesis aventurada afirmar que tal era el esquema con el que

14 "Cada raza, cada época parecen haber tenido una peculiar sensibilidad para determinados valores, y han padecido, en cambio, extraña ceguera para otros. Esto invita a fijar el perfil estimativo de los pueblos y de los grandes períodos históricos. Cada uno se distinguiría por un sistema típico de valoraciones, último secreto de su carácter, de que los acontecimientos serían mera emanación y consecuencia. Asimismo, fuera en extremo interesante estudiar desde este punto de vista las grandes figuras cuya obra ha sido principalmente la invención genial de nuevos valores -así Budha, Cristo, San Francisco de Asís, Maquiavelo, Napoleón. En fin, aquellos otros espíritus soberanos que no han tenido un carácter específico de hombres «prácticos», esto es, de religiosos, moralistas, políticos, pero han descubierto en el universo valores antes latentes: Miguel Ángel, Cervantes, Goya, Dostoyewsky, Stendhal" (III, 549). 
Ortega pudo emprender sus propios estudios sobre los distintos personajes históricos a los que dedicó monografías y biografías, tales como Goethe, Vives, Velázquez o Goya, pues, de hecho, estos mismos aparecen recogidos en el esquema anterior. Queda claro, por tanto, que tal proyecto de investigación hunde sus raíces en la ciencia Estimativa, cuya finalidad no es sino "el examen de las valoraciones humanas, de la evolución de la conciencia de los valores", de ahí el importante recorrido que todo ello tuvo en su obra posterior. "Todo esto -escribía Ortega concluyendo el texto de 1923- y mil atractivas cuestiones más que sugiere la increíble fertilidad del gran tema «Valor» vendrían a componer el pendant histórico a la Estimativa o ciencia a priori del valor, cuyas leyes son de evidencia perfecta, al modo de las geométricas" (III, 549). Podrá discutirse, pues, y creemos que ello deberá ser objeto de futuras investigaciones, en qué puntos concretos se distancia Granell del proyecto orteguiano, en qué tesis y conceptos concretos discrepan, ${ }^{15}$ pero nos resulta difícilmente discutible la intrínseca relación existente entre la Estimativa orteguiana y la Ethología de Granell. Tal es, pues, la fecundísima línea de investigación emprendida por el filósofo ovetense; siempre, empero, reivindicando su condición privilegiada de discípulo de Ortega.

\section{Manuel Granell, una lectura fenomenológica de Ortega}

Como apuntamos al comienzo, Manuel Granell, además de elaborar una obra filosófica propia y original, siempre bajo el magisterio orteguiano, nos ofrece una lectura alternativa de Ortega en la línea de la fenomenología, lectura que solo recientemente, gracias a los trabajos de San Martín, ha sido sistematizada con toda precisión. Sin embargo, hasta donde hemos podido investigar, esta importante contribución ha pasado completamente desapercibida hasta hoy, ya que Granell la presentó, digámoslo así, de un modo discreto y, por supuesto, sin entrar en directa confrontación con sus colegas. Ya sabemos la concepción de la fenomenología que seguían manteniendo Marías y Huéscar a la altura de 1982. Pues bien,

15 Como es sabido, uno de los conceptos centrales en la filosofía orteguiana es el de "creencia", de ahí que Granell insista, ya desde su Lógica (1949), en la importancia de analizar con toda precisión este concepto: "Por consiguiente, si queremos enunciar la más inmediata y primaria relación de hombre y cosas, por fuerza hemos de referirnos a ese contar con las creencias. Este es el básico estrato de la vida, de ese estar el hombre en el mundo, por el cual hombre y mundo quedan trabados en radical conexión. Más allá de esta capa pueden pensarse abstractamente sus elementos ingredientes; pero tales elementos ya no son realidad, sino abstracciones, entes de razón. Por eso, todo meditar que se enfrente directa y audazmente con lo humano debe reducirse ante todo a este básico y radical estrato. Sólo después, una vez aclarado y aprehendido en su mismidad, será posible ascender sin riesgos de extravíos a estratos superiores" (2008, 551-552). Ahora bien, y esto es lo decisivo, como bien advierte Granell, y en ello ha insistido ampliamente Javier San Martín (2008), nuestro primer y más radical anclaje racional en la realidad nos remite, precisamente, a las creencias básicas u originarias, sobre las cuales se fundan y sostienen todas las demás "ideas" y "creencias" (siguiendo la terminología orteguiana). Se torna necesaria, pues, una jerarquización entre las creencias, motivo en el que insiste explícitamente Granell -“Es comprensible de suyo que las creencias implican una jerarquía" (ib., 549)-, así como el reconocimiento, y en esto corrige expresamente a su maestro, de "la existencia de creencias individuales", y no meramente colectivas (ib., 550, nota 10). Por ello, advierte Granell en este punto que "las líneas siguientes se desvían del pensamiento de Ortega" (ib.). Esta corrección nos parece sintomática de la formación fenomenológica de Granell, quien, como ya nos advirtió, había estudiado las Investigaciones lógicas "al dedillo". No estamos, pues, ante un lector "venerativo", por emplear la expresión de Cerezo, sino crítico en el mejor sentido del término, sin que ello le impidiese reconocerse y reivindicarse discípulo de Ortega. 
frente a ella, Granell escribe en 1987 un estudio introductorio de casi cincuenta páginas a $E l$ tema de nuestro tiempo publicado en la editorial Espasa Calpe, ${ }^{16}$ que, hasta donde sabemos, no ha sido tenido en cuenta hasta hoy por los intérpretes de Ortega. Este estudio introductorio tampoco aparece recogido en la citada edición digital que la Fundación Manuel Granell realizó en 2008 de sus obras. Con todo, creemos que estamos ante uno de los estudios más importantes que Granell dedica a su maestro, un escrito de madurez, y de los últimos que escribe, puesto que muere en 1993, ofreciéndonos allí una de las mejores hojas de ruta, si se nos permite la expresión, para abordar el tema de la Estimativa orteguiana, tanto por los textos a los que hace referencia como por las intuiciones esbozadas. Sin poder analizar aquí este escrito con el detenimiento que merece, sí que nos detendremos, al menos, en el siguiente pasaje como muestra fehaciente de lo que pretendemos mostrar:

El racionalista desdeñaba lo corpóreo, y en consecuencia ignoró la «sensación radical ante la vida», que Ortega realza como «fenómeno primario en historia». Es que partía de un peligroso error psicológico, justo por la parte de verdad involucrada, consistente en reducir la sensibilidad al interno alentar. Clausurada en sí misma, la sensibilidad quedó discriminada hasta que Brentano supo definir todos los fenómenos psíquicos por la intencionalidad (de intentio, a su vez de tendere, tender a). La actividad intelectual y la volitiva son muy claramente intencionales, «tienden-a», se «refieren-a». Por ellas está la conciencia abierta al mundo. Y comunica a los otros su pensar, su querer. Lo afectivo, al contrario, parecía recluirse. Es que, en verdad, quien siente se recluye, vive un estado de ánimo, permanece en lo subjetivo. Los sentimientos son inseparables de su acto consciente. Pero esta verdad del sentir no excluye la de su otra cara. Los fenómenos afectivos también son intencionales: no hay amor sin objeto amado. Lo que ocurre es que el sentir amoroso no sólo tiende $a$ su término, sino que retorna para su íntimo goce. El acto intelectual objetiva y permanece neutral. Su intencionalidad es objetivadora. Los sentimientos dan un paso más: se «refieren-a» los objetos para vivirlos, sentirlos, valorarlos. Obsérvese que sin tal retorno, la conciencia sólo sería un espejo del ahí. Gracias, precisamente, al subjetivar y a la íntima reacción ante lo subjetivado, no estamos inermes ante el mundo. Un ejemplo máximo de esa entrañable reacción es el valorar, el estimar valores. Pero, en rigor, todos los actos de conciencia, intelectuales o volitivos, se producen desde la oscura decisión del sentir. Claro que en el sentir cabe distinguir cuatro capas, correspondientes a cuerpo, intracuerpo, psique y espíritu. La conciencia así está abierta al mundo; pero éste, a su vez, en entrega al sentir del hombre (Granell, 2010, 36- 37).

Poco puede añadirse a este bellísimo, al tiempo que preciso y riguroso, análisis fenomenológico de un lúcido Granell que ha cumplido ya, no se olvide, los 81 años. Si, de acuerdo con Ortega, uno de los más acuciantes "temas de nuestro tiempo" es, precisamente, la Crítica y superación del idealismo, según reza el subtítulo del citado libro de Rodríguez Huéscar de 1982, es patente que Granell nos ofrece una vía alternativa a la

16 En lo que sigue citaremos esta "Introducción” por la edición de 2010, pp. 9-56. 
"oficial", incluso rupturista, pues, ciertamente, entre la asunción de que "el tramo final del idealismo", de acuerdo con el primero, "estaba representado por Husserl y su fenomenología", viendo en ella su último "canto de cisne", y la tesis fenomenológica del segundo según la cual "La conciencia así está abierta al mundo; pero éste, a su vez, en entrega al sentir del hombre", existiendo entre ambas tesis un abismo filosófico difícilmente conciliable. Por ello consideramos que Granell nos ofrece una de las claves decisivas a la hora de analizar, como pretendemos aquí, "El legado de Ortega y Gasset en el exilio republicano del 39. Continuidades y rupturas". Para comprender el alcance y la actualidad de esta lectura fenomenológica, merece la pena dedicar un breve comentario a este pasaje a la luz de las propias investigaciones husserlianas.

Así, en este prólogo de Granell encontramos algunas alusiones claras a una temática central de la reflexión en torno a la vida afectiva en la tradición fenomenológica: la intencionalidad de los sentimientos. Esta cuestión no sólo encuentra una concordancia con la estimativa de Ortega, sino que guarda sorprendentes similitudes con el proyecto de la ética y la teoría del valor de Husserl.

Como señala Granell, en realidad es Brentano quien restituye los derechos de la sensibilidad como experiencia de sentido: "Brentano supo definir todos los fenómenos psíquicos por la intencionalidad (de intentio, a su vez de tendere, tener a). La actividad intelectual y la volitiva son muy claramente intencionales, «tienden-a», se «refieren-a». Por ellas está la conciencia abierta al mundo" (ib., 36-37). Aunque Granell solo se refiere de forma explícita en este pasaje a Brentano, iniciador de esta tradición en la psicología descriptiva, para el lector atento de la tradición fenomenológica la descripción de la vida afectiva aquí presentada está en clara consonancia con el pensamiento de Husserl.

De acuerdo con la caracterización de Granell, los fenómenos afectivos no sólo son intencionales, pues "no hay amor sin objeto amado" (ib., 37), sino que entrañan un involucramiento de la subjetividad en la que "el sentir amoroso no sólo tiende a su término, sino que retorna a su íntimo goce" (ib.). Así, la intencionalidad de los afectos, como señala Mariano Crespo en relación al mismo tema en Husserl, "No se trata de la intencionalidad «neutral» característica de los actos dóxicos, sino de un tipo peculiar de intencionalidad en la que el sujeto se ve, por así decir, especialmente «implicado» en su correlato" (Crespo, 2012, 19).

Esta implicación o involucramiento se manifiesta en la forma de una auto-afección en la que el sujeto no solo capta su objeto, sino que lo siente, involucrándose subjetivamente con él. Así, el acto que en Granell se llama fenómeno afectivo de carácter intencional se distingue sutilmente, en la misma vivencia, del íntimo goce que manifiesta en este caso el amor, en la forma de una tesitura sensible, el gozo, que en lenguaje de Husserl se llama sentimientos sensibles. Así, en su ejemplo de Investigaciones Lógicas señala que la alegría ante algo favorable, que es un acto intencional, no comprende solo la nota de intencionalidad como acto ejecutado por el yo o "carácter de acto", sino que, como vivencia concreta, involucra tanto al acto con su respectivo objeto como a la representación enlazada "con una sensación de placer, que es apercibida y localizada como excitación afectiva del sujeto psico-físico sensible y como propiedad objetiva", y luego agrega inmediatamente: "el suceso aparece como recubierto por un resplandor rosado." (Husserl, 1997, 510). Así, el sujeto siente su propio placer y localiza la excitación afectiva en su cuerpo. Al mismo tiempo, apercibe y localiza el goce mismo como relativo a una "propiedad objetiva" a la manera de un "resplan- 
dor”. La metáfora del resplandor o la coloración afectiva no es un ejemplo aislado, aparece en una variedad de ocasiones en contextos muy parecidos. Husserl parece querer decir con ella que la sensación, en este caso de placer, es ella misma la que se apercibe y se localiza tanto en el cuerpo como en el objeto de agrado; aunque en este último, en la forma de una "coloración afectiva" (cfr. Zirión, 2018).

Volviendo a Granell, en el mismo escrito sugiere más adelante un aspecto que para la fenomenología de la vida afectiva es central: "El acto intelectual objetiva y permanece neutral. Su intencionalidad es objetivadora. Los sentimientos dan un paso más: se «refieren a» los objetos para vivirlos, sentirlos, valorarlos" (Granell, 2010, 37). Parece claro que los sentimientos en su referencia intencional a sus objetos imprimen un sello subjetivo que abren, como en Husserl, Scheler y Ortega, a la dimensión del valor: "Obsérvese que, sin tal retorno, la conciencia sólo sería un espejo del ahí. Gracias, precisamente, al subjetivar y a la íntima reacción ante lo subjetivado, no estamos inermes ante el mundo. Un ejemplo máximo de esa entrañable reacción es el valorar, el estimar valores" (ib.)

En diferentes escritos que van desde sus lecciones de Ética y Teoría del valor de 1908 y 1914, pasando por el tratado inédito conocido como Ideas II, hasta sus últimas lecciones de Introducción a la Ética (1920/1924), así como diferentes escritos redactados dentro de este periodo, Husserl sostiene de forma decidida que la vida afectiva, los sentimientos, son la instancia que nos entrega la esfera del valor.

Aunque la mayor parte de estas investigaciones fueron desconocidas más allá del círculo inmediato de asistentes de los cursos de Husserl, así como sus colaboradores cercanos, en la conferencia sobre estimativa de Ortega encontramos, además de alusiones explícitas a las Investigaciones Lógicas (cfr. VII, 716; 717), la noticia de una referencia indirecta a las lecciones de Husserl de 1908 a través del polémico estudio de Theodor Lessing, Estudios sobre Axiomática del valor. El artículo de Lessing retoma, sin dar crédito a Husserl, las líneas centrales de la axiología formal propuesta por su maestro. ${ }^{17}$ Ortega cita de forma explícita el artículo de Lessing en su Discurso de 1918 (cfr. VII, 733, nota 1). Tal y como nos informa Ullrich Melle, editor de las lecciones de Ética y Teoría del Valor de Husserl (1908-1914) (Husserl, 1988, xxiv) Lessing había estudiado con Husserl en el semestre de verano de 1906 y en el de invierno de 1906/07, y en la primavera apareció el citado artículo, publicación por la que Husserl se sintió engañado al considerar que se basaba en su trabajo inédito de investigación. ${ }^{18}$ Sin embargo, no está claro hasta qué punto Ortega supiera el origen de la argumentación central de Lessing. ${ }^{19}$

17 Sobre el asunto de Lessing cfr., además, el estudio de (Baron, 1983) y la referencia explícita del propio Husserl en Lógica Formal y Lógica Trascendental (2009, 192/1974, 142, n.1). Advirtamos, además, que Lessing se refiere explícitamente a Husserl en la edición que cita Ortega, de modo que, aunque es claro que Ortega no sigue a Lessing, y en consecuencia tampoco a Husserl en el énfasis formal de la axiología (cfr. VII, 733, nota 1), sabemos que Ortega conocía el proyecto de Husserl, así sea indirectamente, vía Lessing. Y lo sorprendente es que el mismo Husserl avanzará en su proyecto axiológico en una dirección completamente afín a la Estimativa de Ortega, con clarísimas resonancias, como hemos visto, en la Ethología de Granell.

18 Esto dio lugar a lo que Melle llama el "Lessing-Affäre", ya que Husserl denunció el supuesto plagio, lo cual obligó a Lessing a retractarse y reconocer que su trabajo se inspiraba en las investigaciones inéditas de Husserl (cfr. Melle, ib., xxv-xxvii).

19 Husserl y Ortega tuvieron un estrecho contacto personal años después de aquellas lecciones en Gotinga, y después de las conferencias sobre Estimativa. Aunque, hasta donde sabemos, no se conserva correspondencia entre 
Para Husserl la intencionalidad de los actos afectivos y volitivos es consistente con la estructura del juicio axiológico y práctico (cfr. 1988, 60). La tendencia intencional de los afectos revela la misma estructura de la mención judicativa en su referencia al objeto del juicio, y al eventual cumplimiento de su sentido en una experiencia concordante. Así, el correlato implícito de todo acto afectivo es una forma de valoración, que puede presentarse en la forma de una mera mención o bien a la manera de su cumplimiento en la vivencia gozosa de su exhibición manifiesta en una intuición que la confirma.

De este modo, aunque es claro que para Husserl la vida afectiva es la instancia primordial de acceso al valor, eso no quiere decir que el valor descanse en meras apreciaciones subjetivas, por el contrario, la realización efectiva del valor tiene lugar en síntesis de cumplimiento de orden práctico que involucran no sólo la exhibición afectiva del valor sino una aprobación reflexiva y el compromiso personal, que adquiere la forma de una vocación, con valores que no solo sentimos, sino que asumimos para nuestra vida. De este modo, además de la disposición afectiva, la experiencia de valor, especialmente en el ámbito moral, supone la orientación de la voluntad basada en motivaciones y una decisión libre por un proyecto vital. Base de todas las síntesis de motivación, como sugerirá también Ortega en sus Estudios sobre el amor (V, 455-526), citados por Granell, el amor constituye una tendencia afectiva de fondo que orienta y da sentido a toda motivación de valor. De este modo, de acuerdo con Ortega, el amor constituye un "fondo y soporte al resto de nuestras actividades, cualesquiera que ellas sean"; es más, prosigue,

Nadie vive sin ella, aunque no todos la tienen dentro de sí subrayada con la misma claridad. Contiene nuestra actitud primaria y decisiva ante la realidad total, el sabor que el mundo y la vida tienen para nosotros. El resto de nuestros sentires, pensares, quereres, se mueve ya sobre esa actitud primaria y va montado en ella, coloreado con ella (Ortega, citado por Granell, ib. 40/ V, 503).

En sus últimos años, como lo demuestran investigaciones recientes, así como la publicación póstuma de sus manuscritos tardíos ${ }^{20}$, Husserl tuvo un serio interés por el énfasis en la motivación y la vocación como centro de su reflexión ética (cfr. Peucker, 2008, 322). Ya desde sus lecciones de ética de la década de los años veinte la formación de la persona humana y la auto-constitución moral de la subjetividad adquieren un lugar central (cfr. Melle, 2007, 10; San Martín, 2013b), con sorprendente afinidad tanto con los escritos de Ortega sobre Estimativa, como con el proyecto de la Ethología desarrollada por Granell. Advertimos, pues, la potencialidad de esta lectura fenomenológica del texto orteguiano que nos ofrece Manuel Granell.

ambos filósofos, debemos recordar, y en ello ha insistido San Martín (cfr. 2012, 34), que Ortega visitó a Husserl en Freiburg en 1934, tal y como recoge este último en su correspondencia con Roman Ingarden (cfr. Briefwechsel, III, 298), donde Husserl nos informa de que, según sus palabras, lideraba "una escuela fenomenológica" en España.

20 Cfr. Husserl, 2014, 265-527, especialmente el texto 28, "Vocación individual a la vida en autenticidad personal; vida auténtica como vida en el amor y en el deber absoluto; ser infiel a sí mismo; vida auténtica ante el destino, la enfermedad y la muerte". 


\section{Algunas conclusiones}

Concluyendo, pues, nuestra aproximación a la figura de Manuel Granell, habiendo incidido en su triple condición de filósofo, discípulo y lector de Ortega, no podemos sino insistir en la necesidad de recupear e investigar a fondo la riquísima obra del filósofo ovetense transterrado en Caracas. Estamos, todavía hoy, más de un siglo después de que Ortega la presentara por primera vez en 1916, lejos de haber calibrado mínimamente el alcance de su proyectada ciencia Estimativa, de la que, con toda probabilidad, fuera su gran discípulo, Manuel Granell, quien más rendimiento filosófifico haya sabido extraer, por más que, como él mismo insiste, lo hiciera, "con acento más propio", bajo el proyecto de su Ethología. Por ello hemos querido insistir, igualmente, en el camino fenomenológico que va de la Estimativa a la Ethología, pues también aquí ha sido Granell un discípulo aventajado, sabiendo ofrecernos sutil y perspicazmente las bases fundamentales para una lectura fenomenológica del texto orteguiano, lectura que, gracias a los trabajos de San Martín, ha ido cobrando fuerza frente a la "oficial" y comúnmente asumida por los intérpretes. Estamos, en suma, ante una de las figuras fundamentales del pensamiento filosófico en lengua hispana, que solo por el trágico destino que le tocó vivir a España durante el pasado siglo XX puede explicarse que su nombre y, sobre todo, su filosofía, haya sido injustamente olvidada. Sirvan, pues, estas páginas para contribuir al que esperamos que sea el comienzo de la más que justificada y necesaria recuperación de la figura de Manuel Granell, filósofo, discípulo y lector de Ortega.

\section{Bibliografía}

Abellán, J. L. (1978), Panorama de la filosofía española actual, Madrid: Espasa.

Abellán, J.L. y Mallo, T. (1991), La Escuela de Madrid. Un ensayo de filosofía. Madrid: Asamblea de Madrid.

Acevedo, J. (2015), Ortega y Gasset. ¿Qué significa vivir humanamente?, Santiago de Chile: Editorial Universitaria, pp. 167-186.

Álvarez, L. J.; Hidalgo, A. y Granell, M. (1980), «Entrevista a Manuel Granell», El Basilisco, 11, 1980, pp. 48-56.

Baron, L. (1983), «Discipleship and Dissent: Theodor Lessing and Edmund Husserl» Proceedings of the American Philosophical Society, Vol. 127, 1, pp. 32-49.

Boletín de estudios de filosofía y cultura Manuel Mindán VI, Pensamiento español contemporáneo: La Escuela de Madrid, Calanda: Fundación Mindán Manero.

Crespo, M. (2012), El valor ético de la afectividad, Santiado de Chile: Universidad Católica de Chile.

Díaz Álvarez, J. M. (2009), «Husserl a la altura de los tiempos. Apuntes incompletos para una historia de su recepción en la España contemporánea», en: Boletín de Estudios de Filosofía y Cultura, V, Calanda: Ayuntamiento, pp. 105-122.

Echeverría, J. y García, S. (2017), «La Estimativa de Ortega y sus circunstancias», Revista de Estudios Orteguianos, 34, pp. 81-115.

Expósito Ropero, N. (2019), «Lecturas de Ortega. A propósito de su fenomenología de los valores y su Estimativa», en: Acta Mexicana de Fenomenología. Revista de Investigación filosófica y científica, 4, pp. 57-102. 
Expósito Ropero, N. (2019b), «La Estimativa de Ortega: de sus circunstancias a sus bases fenomenológicas», en: Revista de Estudios Orteguianos, 39, pp. 187-220.

García Gómez, J. (2009), Caminos de la reflexión: la teoría orteguiana de las ideas y las creencias en el contexto de la Escuela de Madrid, Madrid, Biblioteca Nueva-Fundación José Ortega y Gasset.

Gracia, D. (2011), La cuestión del valor, Madrid, Real Academia de Ciencias Morales y Políticas, Discurso de ingreso, 11 de enero de 2011.

Granell, M. (1949), Lógica Moderna, Madrid: Revista de Occidente.

Granell, M. (1969), La vecindad humana. Fundamentación de la Ethología, Madrid: Revista de Occidente.

Granell, M. (1977), Ethología y existencia, Caracas: Equinoccio.

Granell, M. (1983), Humanismo integral (Antología filosófica), Gijón: Noega 1983.

Granell, M. (2008), Obras, Fundación Manuel Granell, ediciónn digital: http://www.fundacionmanuelgranell.com/manuelGranell.swf [visitado por última vez el 21.5.2019].

Granell, M. (2010), «Introducción», en Ortega y Gasset, J., El tema de nuestro tiempo, Madrid: Espasa Calpe, 2010, pp. 9-56.

Husserl, E. (1974), Formale and transzendentale Logik. Versuch einer Kritik der logischen Vernunft. The Hague: Martinus Nijhoff. Edición de Paul Janssen.

Husserl, E. (1975), Logische Untersuchungen. Erster Teil. Prolegomena zur reinen Logik. Text der 1. und der 2. Auflage. Halle: 1900, ed. rev. 1913. The Hague: Martinus Nijhoff. Edición de Elmar Holenstein.

Husserl, E. (1988), Vorlesungen über Ethik und Wertlehre. 1908-1914, The Hague: Kluwer Academic. Edición de Ullrich Melle.

Husserl, E. (1994), Briefwechsel. III. Die Göttinger Schule, en Husserliana Dokumente

III/I-10. Band VIII, The Hage: Kluwer Academic Publishers. Edición de Karl Schuhmann.

Husserl, E. (1997), Investigaciones Lógicas, Madrid: Alianza. Traducción de José Gaos y Manuel García Morente.

Husserl, E. (2004), Einleitung in die Ethik. Vorlesungen Sommersemester 1920 und 1924, Dordrecht: Kluwer Academic. Edición de Henning Peucker.

Husserl, E. (2014), Grenzprobleme der Phänomenologie. Analysen des Unbewusstseins und der Instinkte. Metaphysik. Späte Ethik (Texte aus dem Nachlass 1908 - 1937), New York: Springer. Edición de Rochus Sowa y Thomas Vongehr.

Lasaga, J. (2016), "La Escuela di Madrid: Un'interpretazione", en: Parente, L. (ed.), La Scuola di Madrid. Filosofia spagnola del xx secolo, Milán: Mimesis, pp. 107-124.

Marías, J. (1959), La escuela de Madrid. Estudios de filosofía española, Buenos Aires: Emecé Editores.

Marías, J. (1971), Acera de Ortega, Madrid: Editorial Revista de Occidente.

Marías, J. (1982), "Prólogo”, en: Rodríguez Huésccar, A. (1982), La innovación metafísica de Ortega: crítica y superación del idealismo, Madrid: Ministerio de Educación y Ciencia, pp. 9-14.

Melle, U. (1988), «Einleitung des Herausgebers», en: Husserl, E., Vorlesungen über Ethik und Wertlehre. 1908-1914. Edición de Ullrich Melle, The Hague: Kluwer Academic Publishers, pp. XIII-XLIX. 
Muguerza, J. (2002), «Semblanza de Antonio Rodríguez Huéscar», en: Rodríguez Huéscar, A. (2002), La innovación metafísica de Ortega: crítica y superación del idealismo, Madrid: Biblioteca Nueva, pp.11-16. Edición de Jorge García-Gómez.

Ortega y Gasset, J. (2004-2010), Obras completas, 10 vols., Madrid: Fundación José Ortega y Gasset/ Taurus.

Ortega y Gasset, J. (2016), «Notas de trabajo sobre Estimativa. Primera parte», Revista de Estudios Orteguianos, 32, pp. 5-54. Edición de Javier Echeverría y Dolores Sánchez.

Ortega y Gasset, J. (2016), «Notas de trabajo sobre Estimativa. Segunda parte», Revista de Estudios Orteguianos, 33, pp. 7-25. edición de Javier Echeverría y Dolores Sánchez.

Ortega y Gasset, J. (2017), «Notas de trabajo sobre Estimativa. Tercera parte», Revista de Estudios Orteguianos, 35, pp. 7-36. Edición de Javier Echeverría y Dolores Sánchez.

Padilla, J. (2007), Ortega y Gasset en continuidad. Sobre la Escuela de Madrid. Madrid: Biblioteca Nueva.

Parra Ferreras, J. A. (2015), Ortega y Gasset y el relativismo ético, Tesis Doctoral defendida en la UNED, disponible en e-espacio: http://e-spacio.uned.es/fez/view/tesisuned:FilosofiaJaparra [consultado última vez: 24. 5.19].

Peucker, H. (2008), «From Logic to the Person: An Introduction to Edmund Husserl's Ethics», The Review of Metaphysics, 62, 2, pp. 307-325.

Rodríguez Huéscar, A. (1982), La innovación metafísica de Ortega: crítica y superación del idealismo, Madrid: Ministerio de Educación y Ciencia.

San Martín, J. (2008), «La percepción como interpretación», Investigaciones fenomenológicas 6, pp. 13-32.

San Martín, J. (2012), La fenomenología de Ortega y Gasset, Madrid: Biblioteca Nueva.

San Martín, J. (2013), «La recepción de la fenomenología y su filosofía en torno a El tema de nuestro tiempo», en: Zamora Bonilla J. (ed.), Guía Comares de Ortega y Gasset, Granada: Comares, pp. 47-67.

San Martín, J. (2013b), «Valores y racionalidad en los diversos tipos de cultura», Escritos de Filosofía. Segunda Serie (Buenos Aires, 2013), 1, pp. 113-129.

San Martín, J. (2016), «Epílogo», en: Parente, L. (ed.), La Scuola di Madrid. Filosofia spagnola del xx secolo, Milán: Mimesis, pp. 255-264.

Sánchez Cámara, I. (2010), «Ortega y Gasset y la filosofía de los valores», Revista de Estudios Orteguianos, 1, pp. 159-170.

Scotton, P. (2018), «La formación del ser humano. Sobre el humanismo de Manuel Granell», Res publica, 21.3, pp. 497-515.

Serrano de Haro, A. (2009), «Fenomenología y filosofía primera en perspectiva española», en: Boletín de Estudios de Filosofía y Cultura, V, Calanda: Ayuntamiento, pp. 141-152.

Zirión, A. (2018), «Colorations and moods in Husserl's Studien zur Struktur des Bewusstseins (with a final hint towards the coloring of life)», en: Parker, R. y Quepons, I. (eds.), The New Yearbook for Phenomenology and Phenomenological Philosophy, Vol. XVI, Routledge. 